\title{
Synthèse
}

Pisciculture de quelques espèces

\section{L'élevage de l'esturgeon sibérien (Acipenser baerii Brandt) en France}

Patrick Williot

Cemagref

4, rue du pas de madame 33980 Audenge

France

<williot.patrick@neuf.fr>

\begin{abstract}
Résumé
L'objectif de cet article est de fournir une synthèse des conditions dans lesquelles l'élevage de l'esturgeon sibérien (Acipenser baerii Brandt) a été réalisé en France. Cette espèce a été utilisée comme modèle biologique pour la sauvegarde de l'espèce indigène en danger: l'esturgeon atlantique européen, Acipenser sturio. Successivement sont décrits : les origines de cette activité, les diverses phases de son développement, jusqu'à la situation actuelle, et quelques réflexions en guise de perspective. Un accent particulier est mis sur les actions de recherche.
\end{abstract}

Mots clés : esturgeon sibérien ; France ; pisciculture ; recherche développement.

Thèmes : productions animales ; pêche et aquaculture.

\section{Abstract \\ Siberian sturgeon (Acipenser baerii Brandt) farming in France}

This paper aims at giving a synthesis of the ways by which the farming of the Siberian sturgeon (Acipenser baerii Brandt) occurred in France. The species was primarily a biological model to get experience and methods useful for the safeguard of the endangered European Atlantic sturgeon (Acipenser sturio). The main parts of the paper are the origin of the actions and the different steps of development up to the present status of Siberian sturgeon farming. Special emphasis is given to research.

Key words: development research; France; fish culture; siberian sturgeon.

Subjects: animal productions; fishing and aquaculture. ette synthèse a pour but de décrire les conditions du développement de l'élevage de cette espèce d'esturgeon étrangère en France : pourquoi et comment s'est réalisée cette activité ? Quelles réflexions peut-on formuler pour l'avenir? Dans la présentation, le parti pris est résolument celui de la recherche.

\section{Origine}

Au début des années 1970, à l'occasion d'une enquête générale sur la pêcherie des poissons migrateurs en Gironde (Ctgref, 1973), il était apparu que l'esturgeon atlantique européen, Acipenser sturio, n'était plus l'objet d'une pêche dirigée à cause d'un affaiblissement extrême de sa population.

Très vite, quelques personnes ont refusé le caractère inéluctable de cette évolution, et une stratégie de restauration en trois points a été définie vers la fin des années 1970 (Williot et al. 2004) :

- le soutien de stock, ou repeuplement, sera nécessaire pour aider la population à se restaurer. Cela signifiait qu'il fallait s'orienter vers la maîtrise de la reproduction de géniteurs sauvages capturés en rivière, élever les jeunes jusqu'à quelques grammes pour ensuite les lâcher en rivière à l'image de l'énorme programme de soutien de stock mis en place par les Soviétiques en mer Caspienne et mer d'Azov (Charlon et Williot, 1978) ; 
- un effort devra être consenti pour accroître les connaissances sur l'écologie de cette population, notamment en termes de dynamique;

- enfin, notre inexpérience dans tous les domaines (gestion de géniteurs, reproduction et élevage larvaire principalement) devait être comblée grâce à une autre espèce d'esturgeon comme modèle biologique.

Dans le cadre d'échanges de coopération en océanologie naissant entre la France et l'URSS, des collègues soviétiques vont nous proposer l'esturgeon sibérien, $\mathrm{Aci}$ penser baerii Brandt, qui a l'avantage d'effectuer tout son cycle en eau douce. C'est ainsi que deux arrivages ont été organisés, le premier de 200 individus de $360 \mathrm{~g}$ de poids moyen (intervalle : 50-500 g), en 1975, et le second d'un millier d'alevins de $5 \mathrm{~g}$, en 1982. Dans les deux cas, les alevins provenaient de la reproduction de géniteurs issus de la Léna réalisée dans la pisciculture de Konakova à proximité de Moscou.

Le premier objectif a été d'élever ces animaux. Ils furent nourris avec des aliments composés pour truite et élevés, parfois, dans des conditions non optimales pour le premier lot. Malgré cela, des potentialités de croissance et des performances alimentaires prometteuses ont été mises en évidence à la fin des années 1970 (Barrucand et al., 1978). Ainsi, cette espèce a commencé à présenter un intérêt supplémentaire. Pour qu'une perspective d'élevage se dessine un peu plus, il restait à maîtriser la reproduction et l'élevage larvaire pour s'affranchir des aléas d'un approvisionnement régulier auprès de l'URSS. Cela fut obtenu en 1981 puis en 1982 (Williot et Rouault, 1982 ; Williot et Brun, 1982). Dès lors, l'esturgeon sibérien continua à être un modèle biologique, mais celui-ci présentait désormais aussi un intérêt pour l'élevage.

\section{Développement}

On peut distinguer quatre phases dans le développement de cette production : les prémisses, la phase d'initiation, la première étape et l'inflexion et enfin la phase actuelle de maturité.

\section{Prémisses}

Dès lors que l'espèce modèle présentait son propre potentiel de développement, les actions de recherche privilégiées ont été celles (en plus de celles menées sur l'écologie de la population relique) pouvant être utiles à la fois à la restauration de l'espèce indigène et au développement de l'élevage de l'esturgeon sibérien.

Cela fut notamment le cas durant deux contrats de plan État-Région au cours des années 1980. Plusieurs caractéristiques biologiques des esturgeons ont influencé la définition des axes de recherches, ce sont: une puberté tardive, une absence de dimorphisme sexuel, une ovogenèse pluriannuelle et une incapacité pour les géniteurs à donner naturellement leurs gamètes dès lors qu'ils sont soustraits au milieu naturel.

Ainsi, les principales voies explorées furent la reproduction avec un accent particulier sur la gestion des géniteurs, la gamétogenèse et le sexage précoce, l'alimentation et la nutrition, la pathologie et la cryoconservation du sperme dans une moindre mesure. Pour cela, des coopérations essentiellement nationales furent d'abord mises en œuvre.

Pour ce qui est de la gestion des géniteurs et de leur reproduction stricto sensu, de nombreuses questions devaient être résolues, parmi lesquelles : gestion thermique des géniteurs, modes de gestion d'un stock, choix des meilleurs géniteurs apte à la reproduction, types d'hormone à utiliser. La nécessité d'une vernalisation sera rapidement mise en évidence pour obtenir des gamètes de bonne qualité (Williot et al., 1991). Un peu plus tard seront publiées les données relatives à la gestion d'un stock de génitrices: calendrier d'intervention, critères de présélection et cycles ovariens (Williot et Brun, 1998). L'utilisation du test de compétence à la maturation in vitro des follicules ovariens comme critère de décision pour l'injection hormonale a été initiée (Williot et al., 1991).

En ce qui concerne la gamétogenèse, la fonction femelle sera privilégiée. Sa dynamique sera décrite aux plans histologiques et endocrinologiques (Le Menn et Pelissero, 1991 ; Pelissero et Le Menn, 1991). Il sera nécessaire pour cela de mettre au point plusieurs outils biologiques de dosage des principales hormones ou molécules (vitellogénine) impliquées dans la gamétogenèse (Pelissero, 1988 ; Cuisset et al., 1991). La vitellogénine devait aussi être utilisée comme facteur discriminant des femelles.

Les profils endocriniens permettront de mettre en évidence des similitudes avec ce qui est connu chez les téléostéens et aussi deux faits nouveaux : la production de vitellogénine chez les mâles (Cuisset et al., 1991) et les fortes concentrations en stéroïdes androgènes dans les phases finales de maturation chez les femelles (Pelissero et Le Menn, 1991; Williot, 1997). Pour la première fois, il sera mis en évidence la responsabilité de la nourriture qui contient des substances estrogénomimétiques entraînant la production de vitellogénine par le foie (Pelissero, 1990). Le sexage précoce devra dès lors être réorienté, et ce sera l'hormone stéroïdienne, la 11-cétotestostérone (11-KT), qui montrera un excellent pouvoir discriminant pour les juvéniles d'environ deux ans (Cuisset, 1993). Quant aux teneurs plasmatiques élevées en hormones androgènes chez les femelles matures, leur éventuel rôle physiologique reste inconnu, bien que plusieurs sites de synthèse aient été déterminés pour la 11-KT (Cuisset et al., 1995).

Les premières études sur l'alimentation larvaire (Dabrowski et al., 1985 ; Charlon et Bergot, 1991) ont montré que l'on pouvait obtenir expérimentalement de bons résultats de survie et de croissance avec un sevrage direct sur aliment inerte. En matière de nutrition, il sera montré que le taux de distribution optimal pour des juvéniles se situait autour de 1,45\% du poids vif par jour (Kaushik et al., 1989), que le taux optimal de protéine dans l'aliment était de 40 \% (Kaushik et al., 1991) et, enfin, que l'ingestion volontaire d'aliment diminuait avec l'âge, alors que simultanément l'efficacité de rétention énergétique augmentait (Médale et Kaushik, 1991).

La cryoconservation du sperme sera difficile à mettre en œuvre. En revanche, un premier travail sur la physiologie du sperme mettra en évidence l'influence du potassium sur la motilité (Gallis et al., 1991).

À côté de ces travaux de recherche finalisée, une patiente accumulation de résultats et de mesures conjuguées à une analyse de la bibliographie disponible a conduit à publier une première synthèse des conditions requises pour le grossissement, notamment vis-à-vis de la gestion de l'eau, en particulier de l'oxygène, et de l'alimentation (Williot et al., 1988).

\section{Phase d'initiation}

C'est au cours de la décennie 1980 que divers essais de croissance furent conduits dans diverses piscicultures aux conditions thermiques variées avec un 
strict contrôle sanitaire. Il a été montré que plus la température était faible, moins bonne était la croissance, et aucune pathologie spécifique n'a été observée (Brun et al., 1991). C'est aussi durant cette période que le Cemagref produira et vendra des alevins à des pisciculteurs, afin qu'ils testent la faisabilité de son élevage.

Depuis le début des années 1980, suite à un accord entre l'Inra et le Cemagref, ce dernier avait installé une petite unité expérimentale dans la pisciculture Inra de Donzacq dans le sud des Landes.

Au cours des années 1980, il est apparu souhaitable de sécuriser le stock, et donc le répartir sur un autre site. Ainsi, une partie de celui-ci fut placée dans une pisciculture produisant des truites en Gironde. Cela a été l'occasion pour le pisciculteur de bénéficier de nombreux transferts de savoir-faire et, ainsi, d'initier le démarrage de ce nouvel élevage. Malheureusement, cette pisciculture sera l'objet d'une pollution qui atteindra aussi une partie du stock d'esturgeon.

Les conditions expérimentales de la petite structure expérimentale du Cemagref à Donzacq sont vite apparues contraignantes. Une nouvelle structure propre au Cemagref était nécessaire pour poursuivre les investigations à la fois sur le programme de restauration de l'espèce indigène et sur l'esturgeon sibérien. Dans la seconde moitié des années 1980, la direction générale du Cemagref avait finalement accepté de nous donner son accord pour ce projet, sous réserve que simultanément une société privée s'installe dans le but de produire des esturgeons sibériens. Sur l'initiative du Cemagref, plusieurs réunions furent alors organisées avec tous les intéressés potentiels, éleveurs, fabricants d'aliments, bureaux d'étude et autres industriels. Le contexte du Cemagref et l'ensemble des données biotechniques sur l'élevage ont alors été présentés aux participants. Durant cette phase de maturation, deux autres actions furent conduites, l'une consistait à mener une recherche de site sur la base d'un cahier des charges établi par le Cemagref, qui avait déjà conduit quelques années auparavant un travail exploratoire, et l'autre à faire une étude de marché. Ces deux études avaient été confiées à deux bureaux d'études sur appel d'offres. À la suite de l'étude de marché, les perspectives de commercialisation semblaient séduisantes, et les recommandations étaient de situer l'esturgeon parmi les poissons de haut de gamme. Cela signi- fiait que les prix de vente escomptés étaient élevés, donc que les coûts de production pouvaient aussi être élevés, ce qui permettait d'envisager des modes de production coûteux, et donc la mise en valeur de sites difficiles. Une autre conclusion de cette étude suggérait que le volume d'un tel marché était de l'ordre de quelques milliers de tonne, ce qui laissait entrevoir des perspectives de développement.

Quant à l'étude de site, elle avait permis de mettre en évidence quelques sites intéressants, notamment l'un d'entre eux, car la commune sur laquelle il était situé se proposait de l'acheter et de le mettre à disposition par bail emphytéotique, ce qui réduisait le coût d'investissement.

Sur la base de tous ces éléments, un bureau d'étude spécialisé en aquaculture, avec quelques autres acteurs, décida de créer une société destinée à produire des alevins, la Société anonyme écloserie de Guyenne (SAEG).

Le montage du projet était donc le suivant. Le Cemagref acceptait de se doter de sa propre structure expérimentale, le CREA (Centre de recherche et d'étude sur les acipenséridés, en clin d'œil à l'appellation locale de l'esturgeon indigène, le Creac) dimensionné pour poursuivre aussi les travaux sur l'espèce indigène A. sturio. Juste à côté s'installait l'écloserie privée (SAEG). Les prises d'eau de surface (bras de rivière sur l'Isle, affluent rive droite de la Dordogne) et le forage (eau à température constante de quelques $17-18{ }^{\circ} \mathrm{C}$ de qualité alimentaire) étaient partagés entre les deux structures afin de limiter les coûts. L'investissement initial pour le Cemagref était couvert par : des fonds "Recherche" dans le cadre d'un transfert de savoir faire public-privé, la vente à la SAEG d'un lot important de géniteurs sexés et marqués, la formation de deux ingénieurs, l'assistance technique à la SAEG, surtout durant la première année, une aide financière du ministère de l'Agriculture et du Cemagref.

\section{Première étape et inflexion}

La mise en eau des deux structures a eu lieu en début 1991, et cela peut être considéré comme le début de la première étape dans le développement de cette nouvelle production piscicole.

Elle était fondée sur la production de chair à partir d'animaux pesant entre 3 et $4 \mathrm{~kg}$, c'est-à-dire après environ trois ans d'élevage.
L'écloserie industrielle (SAEG) avait été dimensionnée sur la base des résultats de l'étude de marché. Le potentiel initial de production d'alevins était de quelque 200000 individus avec une seconde phase pouvant accroître sa capacité jusqu'aux environs de 700000 alevins.

La crise du marché du poisson du début des années 1990 a tiré tous les prix vers le bas, de telle sorte que le prix de vente réel des premiers esturgeons était notablement inférieur à ceux escomptés. Ainsi, les entreprises, qui avaient à la fois beaucoup investi, en espérant des prix de vente élevés, et sous-estimé les difficultés de commercialisation d'une espèce par ailleurs méconnue, se sont retrouvées dans une situation délicate. Cette nouvelle situation a aussi compromis définitivement des projets destinés à potentiellement mettre en valeur des sites attractifs par la disponibilité en eaux réchauffées mais nécessitant une ingénierie compliquée, donc coûteuse d'autant qu'aucun engagement de fourniture régulière d'eaux réchauffées ne pouvait être obtenu. En conséquence, l'écloserie commerciale n'a pas vu la demande en alevins se développer comme prévu, et ainsi, s'est retrouvée dans une situation difficile. En cascade, le Cemagref n'a perçu aucune des royalties escomptées et a dû faire face à une situation imprévue pour financer les travaux sur la sauvegarde de l'espèce indigène non soutenus par ailleurs au début des années 1990.

Devant cette nouvelle situation difficile sur le marché de la chair, l'ensemble des acteurs de la filière s'est orienté vers la production de caviar dans la seconde moitié des années 1990. Cette nouvelle orientation était encouragée par le déclin des pêcheries d'esturgeons, notamment dans la principale zone de production, le bassin Ponto-Caspien, et donc l'offre officielle en caviar diminuait.

Cette réorientation comportait au moins deux nouvelles difficultés. Le cycle de production est beaucoup plus long (environ sept ans minimum) et génère une grosse demande en fonds de roulement. La seconde tient au produit lui-même. Aucune image de marque n'était attachée à l'esturgeon sibérien, contrairement aux trois espèces du bassin Ponto-Caspien, le beluga (Huso huso), l'ocietra (Acipenser gueldenstaedtii) et le sevruga (Acipenser stellatus). Cela motivera les producteurs à solliciter une modification de la législation française qui limitait l'autorisation à l'esturgeon sibérien, l'élevage d'esturgeons non indigènes. La nécessité de 
mobiliser des fonds supplémentaires, ajoutée aux autres complications évoquées, entraînera souvent une modification de la structure financière des entreprises.

Au plan de la recherche, plusieurs approches ont été soit approfondies (reproduction), soit développées (ontogenèse précoce, stress hypoxique, tests de toxicités). En ce qui concerne la reproduction, il a été montré que plusieurs types d'hormones étaient utilisables indifféremment chez les deux sexes (Goncharov et al., 2001 ; Williot et al., 2002). Cela était nécessaire pour consolider certains résultats antérieurs, car la présence du GnRH mammalien avait été mise en évidence dans le cerveau de l'esturgeon sibérien (Leprêtre et al., 1993), ce qui ouvrait définitivement la voie à l'usage de cette neurohormone. Un travail méthodologique a permis de fixer les conditions de réalisation du test de compétence à la maturation in vitro des follicules ovariens, utilisé comme critère de choix des meilleures femelles aptes à la reproduction (Williot, 1997). Enfin, la gestion des mâles, depuis l'appréciation de leur degré de maturation sexuelle jusqu'à la motilité du sperme, a permis de dégager les meilleures modalités de leur gestion (Williot et al., 2000). L'ensemble des résultats relatifs à la reproduction a donné lieu à une synthèse publiée en 2002 (Williot, 2002). L'ontogenèse précoce a fait l'objet de plusieurs études avec, en particulier, une description des diverses étapes comportementales jusqu'après la première prise de nourriture, les conditions de celle-ci étant précisées (Gisbert et Williot, 1997). Le développement du tractus digestif durant les premières semaines sera abordé simultanément sous l'angle descriptif (histologie) et fonctionnel (enzymologie) (Gisbert et al., 1998 ; Gisbert et al., 1999), et l'ensemble des données acquises sur l'élevage larvaire donnera lieu à une synthèse (Gisbert et Williot, 2002). L'étude des conséquences d'un stress hypoxique sera menée sous diverses approches (Nonnotte et al., 1993; Maxime et al., 1995). Outre les valeurs de seuil vis-à-vis de l'oxygène, les mécanismes de régulation ont été décrits grâce à des indicateurs plasmatiques du stress validant l'usage de ceuxci pour l'étude d'autres formes de stress. Enfin, on peut signaler la réalisation de tests de sensibilités à l'ammoniaque (Salin et Williot, 1991) et aux nitrites (Huertas et al., 2002) qui permettent de situer l'esturgeon sibérien parmi les espèces assez résistantes à ces molécules.

\section{Situation actuelle}

L'objectif essentiel de la production est le caviar. Le schéma de production a été décrit (Williot et Sabeau, 1999), il consiste à sexer les animaux vers trois ans, vendre les mâles, puis poursuivre l'élevage des femelles jusqu'à obtenir le développement quasi complet des ovaires.

Le sexage est effectué par échographie, ce qui permet de discriminer le sexe des animaux à partir de trois ans avec une bonne efficacité et une grande rapidité.

Les femelles sont alors élevées dans des structures qui peuvent être des raceways, des bassins en terre plus ou moins grands $\left(500\right.$ à $5000 \mathrm{~m}^{2}$ ). Le taux de distribution de la nourriture (aliments granulés) est en général peu élevé durant cette phase (le plus souvent inférieur à 1).

Durant la période de grossissement, certains producteurs effectuent un tri sur la base de la taille des follicules ovariens afin de mieux prévoir leur potentiel de production dans le temps. Un dernier contrôle a lieu peu avant la période optimale de préparation du caviar, c'est-à-dire à l'automne. Lorsque la taille des follicules ovariens est jugée homogène et suffisamment développée, les animaux sont alors mis à jeun dans une eau claire afin de se débarrasser si besoin de goûts dits "de terre".

Les animaux sont alors abattus puis les ovaires sont traités selon le procédé classique et bien connu, tamisage, pesée, contrôle de texture et de goût de façon à classer les produits selon leur qualité, puis salage, égouttage, mise en boîte et fermeture. Durant toutes ces opérations, les lots sont suivis par les numéros des femelles, ainsi la traçabilité est complète.

Il y a actuellement quatre acteurs commerciaux principaux, chacun pouvant gérer un ou plusieurs sites de grossissement. Les sites sont essentiellement localisés dans le grand Sud-Ouest de la France. On observe une tendance à l'intégration verticale, de la gestion de la reproduction à la vente de produits finis. Aussi apparaissent des structures de productions qui cèdent leurs femelles à des acteurs spécialisés.

La production française de caviar est passée de 3,5 tonnes/an en 1999, à environ 20 tonnes en 2007. C'est la plus importante pour ce qui concerne l'esturgeon sibérien. Cette évolution est très proche de celle anticipée à la fin des années 1990 (Beyer, 1999). Elle est en augmentation à peu près régulière depuis plusieurs années. Globalement, la production fran- çaise de caviar se situe avec l'Italie dans le peloton de tête des pays producteurs au plan quantitatif.

\section{Perspectives}

La plupart des élevages français ont acquis une grande compétence qui se traduit par une grande qualité de leurs produits.

Quels sont les principaux éléments de réflexion? Ce sont le marché, la concurrence, les conditions de production, la diversification et une organisation professionnelle.

La situation des pêcheries en Eurasie n'a cessé de se dégrader (Williot et al., 2002) à tel point qu'il est prévisible que le caviar issu d'esturgeon d'élevage va se substituer totalement à celui d'origine sauvage dans les prochaines années. Le marché donnant lieu à des flux internationaux avait été chiffré à quelques centaines de tonnes à la fin de la période faste (Williot et Bourguignon, 1991). Depuis, il s'est restreint, car l'offre a diminué, mais aussi, la demande. Nombreuses ont été, par exemple, les compagnies aériennes qui avaient supprimé leurs approvisionnements pour réduire leurs coûts. Cette situation est en train de se renverser. On peut penser en outre que la demande va augmenter de la part de nouveaux pays émergents. Ainsi, on peut raisonnablement prédire un marché mondial (celui donnant lieu à des flux) du même ordre de grandeur que celui existant auparavant, soit de quelques centaines de tonnes.

La concurrence apparaîtra avec l'augmentation de la production qui pourra se faire à partir de plusieurs espèces et entre pays. Pour ce qui est de l'esturgeon sibérien par exemple, cette espèce est présente dans de très nombreux pays depuis la Chine, l'Uruguay, les EtatsUnis (Floride) et l'Europe Centrale avec des stocks croissants (plus de 500 tonnes en Pologne), et des productions de caviar non négligeables sont attendues dans les prochaines années. Dans ces pays, de nouvelles fermes sont actuellement en cours de construction ou de mise en service, et cela en mobilisant le Fonds européen pour la pêche (FEP). La production de caviar à partir de l'élevage des fameuses trois espèces du bassin PontoCaspien est encore négligeable, même si quelques centaines de kilogrammes de caviar ont déjà été obtenues en Bulgarie. Mais d'autres espèces que l'esturgeon sibérien sans image commerciale forte sont déjà des acteurs du marché. On 
peut citer l'esturgeon blanc (Acipenser transmontanus), originaire des côtes américaines du nord pacifique, et l'esturgeon adriatique (Acipenser naccarii), tous deux l'objet d'élevage en Europe. Ainsi, la diversification de l'offre par les espèces augmentera probablement surtout si la législation sur les espèces s'assouplit comme cela vient d'être le cas en France. Les éleveurs français ne seront plus en état de concurrence tronquée vis-à-vis de la plupart de leurs confrères européens (Williot et al., 1993).

Les conditions de production touchent l'accès à l'eau (eau douce) et à la législation sur les normes de rejet. La concurrence avec les autres usages est déjà parfois très forte. Ceux qui auront acquis la maitrise de ce facteur de production sans répercussion négative sur la qualité s'assureront un avantage certain sur le long terme. L'une des principales difficultés de cette production est de tester l'impact de modifications de gestion, dont les effets sont très différés dans le temps. Il y a là un champ de réflexion et d'investigation vierge. La connaissance (une caractérisation) des stocks et une gestion appropriée des reproductions sont probablement un gage de sécurité de fonctionnement sur le long terme. Une recherche de la diversification des espèces (déjà évoquée plus haut en partie) à cycle court, tels que sterlet (Acipenser ruthenus), sera peut-être à envisager. On peut aussi imaginer une recherche dans la diversification des produits par leur nature, comme, par exemple, les travaux menés en Russie, en Pologne et en Allemagne sur le traitement non plus de follicules ovariens mais d'œufs ovulés. L'avantage est de sauvegarder la femelle, donc de réduire les coûts, mais alors le produit est et, probablement, restera différent du caviar traditionnel. Un autre moyen, peut-être de réduire les coûts, serait de ne produire que des femelles. Bien que le déterminisme du sexe soit inconnu (Wuertz et al., 2006), un très récent travail a mis en évidence la production d'animaux gynogénétiques (Foop-Bayat, 2007). Des approches économiques devraient compléter ces divers objectifs.

Les questions d'appellation des produits, peut-être des zones de production ou des types de productions, se feront probablement jour à l'image de ce qui existe dans le reste des productions agricoles. Et dans cette voie, une organisation professionnelle forte doit être encouragée.

\section{Remerciements}

Ce travail fournit l'occasion de remercier quelques personnes dont le soutien fut déterminant au démarrage de ce programme ; il s'agit de Pierre Lamarque, Philippe Ferlin et Pierre Luquet. Ensuite, ce programme a bénéficié du concours actif de Thierry Rouault, Rémi Brun, puis de Jacques Bories. Enfin, René Lésel doit être remercié pour avoir fourni l'occasion de cette synthèse.

\section{Références}

Barrucand $\mathrm{M}$, Ferlin $\mathrm{P}$, Lamarque $\mathrm{P}$, Sabaut JJ. Alimentation artificielle de l'esturgeon Acipenser baerii. Proceeding of World Symposium on Finfish Nutrition and Fishfeed Technology, Hamburg, 1978.

Beyer O. Les perspectives des marchés de caviar et d'esturgeons d'élevage. CR Acad Agric Fr 1999; 85 : 105-18.

Brun R, Nougayrede P, Chene P, Vuillaume A, Crespeau F. Bilan sanitaire de deux ans d'élevage d'Acipenser baerii en piscicultures intensives. In: Williot P, ed. Acipenser. Antony (France) : Cemagref Publication, 1991.

Charlon N, Bergot P. Alimentation artificielle des larves de l'esturgeon sibérien (Acipensrer baerii Brandt). In : Williot $\mathrm{P}$, ed. Acipenser. Antony (France): Cemagref Publication, 1991.

Charlon N, Williot P. Élevage d'esturgeons de repeuplement et de consommation en URSS. Bull Centre Etud Rech Sci Biarritz 1978; 12: 7-156.

CTGREF. Rapport sur la pêche en Gironde. SI : CTGREF, 1973.

Cuisset B, Fostier A, Williot P, BenneteauPelissero $C$, Le Menn F. Occurrence and in vitro biosynthesis of 11-ketotestosterone in Siberian sturgeon, Acipenser baerii Brandt maturing females. Fish Physiol Biochem 1995 ; $14:$ 313-22.

Cuisset B, Pelissero C, Le Menn F, Nunez Rodriguez J. Elisa for Siberian sturgeon (Acipenser baerii) vitellogenine. In : Williot $\mathrm{P}$, ed. Acipenser. Antony (France): Cemagref Publication, 1991.

Cuisset B. Étude endocrinologique de la fonction de reproduction chez l'esturgeon sibérien Acipenser baerii Brandt : application au sexage des populations sauvages ou élevées en Acipensériculture. Thèse $\mathrm{n}^{\circ} 1039$, université Bordeaux-I, 1993.

Dabrowski K, Kaushik S, Fauconneau B. Rearing of sturgeon (Acipenser baerii Brandt) larvae. I. Feeding trial. Aquaculture 1985; 47 : 185-92.

Foop-Bayat D. Verification of meiotic gynogenesis in Siberian sturgeon (Acipenser baerii) using microsatellites DNA and cytogenetical markers. J Fish Biol 2007; 71: 478-85.

Gallis JL, Fedrigo E, Jatteau P, Bonpunt E, Billard R. Siberian sturgeon, Acipenser baerii, spermatozoa: effects of dilution, $\mathrm{pH}$, osmotic pressure, sodium and potassium ions on motility. In: Williot $\mathrm{P}$, ed. Acipenser. Antony (France) : Cemagref'Publication, 1991.
Gisbert E, Rodriguez A, Castellò-Orvay F, Williot P. A histological study of the development of the digestive tract of Siberian sturgeon (Acipenser baerii Brandt) during early ontogeny. Aquaculture 1998; 167 : 195-209.

Gisbert E, Sarasquette MC, Williot P, CastelloOrvay F. Histochemistry of the development of the digestive system of Siberian sturgeon during early ontogeny. J Fish Biol 1999; 55 : 596-616.

Gisbert E, Williot P. Larval behaviour and effect of the timing of initial feeding on growth and survival of Siberian sturgeon larvae under small-scale hatchery production. Aquaculture $1997 ; 156: 63-76$.

Gisbert E, Williot P. Advances in the larval rearing of Siberian sturgeon. J Fish Biol 2002; 60 : 1071-92.

Goncharov B, Williot P, Le Menn F. Comparison of the effects of gonadotropic preparations of the carp and stellate sturgeon pituitaries on in vivo and in vitro oocyte maturation in the Siberian sturgeon Acipenser baerii Brandt. Rus J Develop Biol 2001; 32 : 320-7.

Huertas M, Gisbert E, Rodriguez A, Cardona L, Williot $P$, Castello-Orvay F. Acute exposure of Siberian sturgeon (Acipenser baerii Brandt) to nitrite: median lethal concentration (LC50) determination, hematological changes and nitrite accumulation in selected tissues. Aquat Toxicol 2002 ; 57 : 257-66.

Kaushik S, Breque J, Blanc D. Requirements for protein and essential amino acids and their utilization by Siberian sturgeon (Acipenser baerii). In : Williot P, ed. Acipenser. Antony (France) : Cemagref Publication, 1991.

Kaushik S, Luquet $P$, Blanc D, Paba A. Studies on the nutrition of the Siberian sturgeon (Acipenser baerii). I. Utilization of digestible carbohydrates by sturgeon. Aquaculture 1989; 76 : 97-107.

Le Menn F, Pelissero C. Histological and ultrastructural studies of oogenesis of the Siberian sturgeon Acipenser baerii. In: Williot $\mathrm{P}$, ed. Acipenser. Antony (France): Cemagref Publication, 1991

Leprêtre $E$, Anglade I, Williot $P$, Vandesande $F$. Tramu G, Kah O. Comparative distribution of Mammalian GnRH (gonadotrophin-releasing hormone) and Chick. GnRH-II in the brain of the immature Siberian Sturgeon (Acipenser baerii). J Compar Neurol $1993 ; 336$ : 2-17.

Maxime V, Nonnotte G, Peyraud C, Williot P, Truchot JP. Circulatory and respiratory effects of an hypoxic stress in the Siberian sturgeon. Respir Physiol 1995; 100 : 203-12.

Médale F, Kaushik S. Energy utilization by farmed Siberian sturgeon (Acipenser baerii) from 3 age classes. In: Williot P, ed. Acipenser. Antony (France) : Cemagref Publication, 1991.

Nonnotte G, Maxime V, Truchot JP, Williot P, Peyraud C. Respiratory responses to progressive ambient hypoxia in the sturgeon, Acipenser baerii. Respir Physiol 1993 ; 91 : 71-82.

Pelissero C, Le Menn F. Evolution of sex steroid levels in males and first time maturing females of the Siberian sturgeon (Acipenser baerii) reared in a French fish farm. In : Williot $\mathrm{P}$, ed. Acipenser. Antony (France) : Cemagref Publication, 1991.

Pelissero C. Interaction de I'alimentation sur la présence de stéroïdes, d'isoflavones et de dérivés, dans le plasma de l'esturgeon sibérien (Acipenser baerii). Conséquences sur la vitellogenèse. Thèse $n^{\circ} 427$, université Bordeaux-I. 1990. 
Pelissero C. Mise en place des bases méthodo logiques pour l'étude de la reproduction chez l'esturgeon Acipenser baerii femelle. Thèse $\mathrm{n}^{\circ} 2229$, université Bordeaux-I. 1988.

Salin D, Williot P. Acute toxicity of ammonia to Siberian sturgeon, Acipenser baerii B. In : Williot $\mathrm{P}$, ed. Acipenser. Antony (France) : Cemagref Publication, 1991.

Williot P, Arlati G, Chebanov M, et al. Status and management of Eurasian sturgeon: an overview. Intern Rev Hydrobiol 2002; 87: 483-506.

Williot P, Bourguignon G. Production d'estur geons et de caviar, état actuel et perspectives. In: Williot P, ed. Acipenser. Antony (France) Cemagref Publication, 1991.

Williot P, Bronzi P, Arlati G. A very brief survey of status and prospects of freshwater sturgeon farming in Europe (EEC). In : Kestemont P, Billard R, eds. Aquaculture of freshwater species (except salmonids). SI : European Aquaculture Society, 1993.

Williot P, Brun R, Rouault T, Rooryck O. Management of female breeders of the Siberian sturgeon, Acipenser baerii Brandt: first results. In : Williot P, ed. Acipenser. Antony (France) : Cemagref Publication, 1991.
Williot $\mathrm{P}$, Brun $\mathrm{R}$. Résultats sur la reproduction d'Acipenser baerii en 1982. Bull Fr Piscic 1982 ; 287: 19-22.

Williot P, Brun R. Ovarian development and cycles in cultured Siberian sturgeon, Acipenser baerii. Aquat Living Ressour 1998; 11: 111-8.

Williot P, Gulyas T, Ceapa C. An analogue of $\mathrm{GnRH}$ is effective for the induction of ovulation and spermiation in farmed Siberian sturgeon Acipenser baerii Brandt. Aquac Res 2002 ; 33 : 735-7.

Williot P, Kopeika EF, Goncharov B. Influence of testis state, temperature and delay in semen collection on spermatozoa motility in the culture Siberian sturgeon (Acipenser baerii Brandt). Aquaculture 2000 ; 189 : 53-61.

Williot P, Rouault T, Brun R, Miossec G, Rooryck $O$. Grossissement intensif de l'esturgeon sibérien Acipenser baerii en bassin. Aquac Rev $1988 ; 17-18: 29-32 ; 27-32$.

Williot P, Rouault $\mathrm{T}$, Rochard $\mathrm{E}$, et al. French attempts to protect and restore Acipenser sturio in the Gironde: status and perspectives, the research point of view. In: Gessner J, Ritterhoff J, eds. Bundesamt für Naturschutz 2004 . (101) : 83-99.
Williot $P$, Rouault T. Compte rendu d'une première reproduction en France de I'esturgeon sibérien Acipenser baerii. Bull Fr Piscic 1982 ; 286 : 255-61.

Williot P, Sabeau L. Élevage d'esturgeons et production de caviar: exemple de I'esturgeon sibérien (Acipenser baerii) en France. CR Acad Agric 1999 ; 85 : 71-83.

Williot P. Effects of incubation media on maturation of isolated ovarian follicles of Siberian sturgeon (Acipenser baerii Brandt) induced by sturgeon gonadotropic preparation or $17 \alpha$, $20 \beta$, dihydroxy progesterone. Compar Biochem Physiol 1997; 118 C : 285-93.

Williot P. Reproduction de I'esturgeon sibérien (Acipenser baerii Brandt) en élevage: gestion des génitrices, compétence à la maturation in vitro de follicules ovariens et caractéristiques plasmatiques durant l'induction de la ponte. Thèse $\mathrm{n}^{\circ} 1822$, université Bordeaux-I, 1997

Williot P. Reproduction des esturgeons. In Billard R, ed. Esturgeons et caviar. Paris: Lavoisier Tech et Doc, 2002.

Wuertz S, Gaillard S, Barbisan F, et al. Extensive screening of sturgeon genomes by random screening techniques revealed no sexspecific marker. Aquaculture 2006; 258 : $685-8$. 